\title{
Modelos de treinamento de cateterismo vesical feminino e sua importância para a prática médica: uma revisão integrativa da literatura
}

\author{
Training models of female urinary catheterization and \\ their importance for the medical skills: an integrative \\ review of literature
}

\begin{abstract}
Lêda Lima da Silva', Alyne Condurú dos Santos Cunha', Alan Luz Tembra', Geovanna Mourão Pantoja', Mauro de Souza Pantoja', Charles Alberto Villacorta de Barros'

'Laboratório de Morfofisiologia Aplicada à Saúde e Laboratório de Cirurgia Experimental, Universidade do Estado do Pará, Belém (PA), Brasil.
\end{abstract}

\begin{abstract}
RESUMO
Objetivo: Apresentar e analisar o desenvolvimento dos modelos de treinamento de cateterismo vesical feminino e seus benefícios para o aperfeiçoamento das habilidades médicas. Método: revisão integrativa realizada entre novembro de 2017 e janeiro de 2018, com período da busca entre 2000 a 2017, dos artigos indexados no PubMed e na Biblioteca Virtual em Saúde (BVS), a partir das bases de dados SciELO, Medline e a Lilacs. Foram utilizados os Descritores das Ciências da Saúde (DeCS) nas línguas portuguesa, inglesa e espanhola: Cateterismo urinário (Urinary atheterization; Cateterismo urinario), Feminino (female; femenino), Treinamento por simulação (Simulation training; Entrenamiento simulado) e Educação médica (Education, Medical; Educación Médica), associados com o operador booleano "AND", resultado em uma amostra final de 9 artigos. Estes foram analisados por dois pesquisadores independentes. Resultados e Discussão: as evidências possibilitaram a organização de 4 categorias temáticas: simulação em manequim low-tech, simulação em manequim high-tech, grau de confiança e modelo porcino de simulação. Diante disso, a implementação da simulação na grade curricular, bem como a prática repetitiva, com feedback e ambiente clínico representativo da realidade proporcionam um aumento do grau de confiança, das habilidades processuais e uma diminuição da curva de aprendizagem. Conclusão: identifica-se a necessidade de desenvolvimento de modelos de treinamento de baixo custo, uma maior produção científica acerca da sondagem vesical feminina e o envolvimento das instituições para a melhora dos cuidados e da formação de profissionais em prol da saúde da população.
\end{abstract}

\section{COMO CITAR ESTE ARTIGO}

Silva LL, Cunha ACS, Tembra AL, Pantoja GM, Pantoja MS, Barros CAV. Modelos de treinamento de cateterismo vesical feminino e sua importância para a prática médica: uma revisão integrativa da literatura. Interdisciplinary Journal of Health Education. 2019 Jan-Dez;4(1-2):42-48. https://doi.org/10.4322/ijhe.2018.011

\section{CORRESPONDÊNCIA}

Alyne Condurú dos Santos Cunha Universidade do Estado do Pará Travessa Perebebui, 2623, $N$ CEP 66 08 CEP 66087-662, Belém (PA), Brasi alynecunhauepa@hotmail.com

\section{FONTE DE FINANCIAMENTO} Nenhuma.

\section{CONFLITO DE INTERESSE}

Os autores declararam não

haver conflitos de interesse.

O estudo foi realizado em Belém, Pará, Brasil.

Todos os autores leram e aprovam a versão final submetida ao Interdisciplinary Journal of Health Education (IJHE).
PALAVRAS-CHAVE: Treinamento por simulação. Cateterismo urinário. Mulheres. Educação médica.

\begin{abstract}
Objective: To present and analyze the development of female urinary catheterization training models and its benefits for the improvement of medical skills. Method: an integrative review done between November 2017 and January 2018, with a search period between 2000 and 2017, of the articles indexed in PubMed and in Virtual Health Library, from the SciELO, Medline and Lilacs databases. The Health Sciences Descriptors (DeCS) were used in the Portuguese, English and Spanish languages: Urinary catheterization, Feminine, Simulation training and Medical Education, associated with the Boolean operator "AND", resulting in a final sample of 9 articles, which were analyzed by two independent researchers. Results: Evidence allowed the organization of 4 thematic categories: low-tech mannequin simulation, high-tech mannequin simulation, confidence level and porcine simulation model. Discussion: Therefore, the implementation of curricular simulation, as well as repetitive practice, with feedback and a clinical environment representative of reality, provide an increase in the degree of confidence, procedural skills and a decrease in the learning curve. Conclusion: Considering the need to develop low-cost training models, greater scientific production on female urinary catheterization, the involvement of institutions in improving care and training of health professionals for the population must be pursued.
\end{abstract}

KEYWORDS: Simulation training. Urinary catheterization. Female. Education, Medical 


\section{Introdução}

Os modelos de treinamento ou simuladores consistem em objetos físicos e representativos de uma parte ou totalidade do corpo humano, enquanto que a simulação se refere à utilização ativa e controlada destes instrumentos por tecnologias de alta ou baixa complexidades, high-tech elow-tech, respectivamente ${ }^{1,2}$. Nesse interim, inicialmente utilizados na área da aviação, expandiram-se para a área médica na década de 60, com o intuito de possibilitar uma quebra do padrão mecânico e biológico, a partir da integração entre os conteúdos teóricos e práticos ${ }^{1-4}$.

Historicamente, no início da década de 60, o primeiro modelo de treinamento médico foi desenvolvido por Asmund Laerdal, na Noruega, com o modelo denominado Resusci-Anne, utilizado para o treinamento de respiração boca-a-boca e, posteriormente adaptado para a prática de situações de urgência e emergência, como o Suporte Básico de Vida e a Ressuscitação Cardiopulmonar. Em seguida, o modelo Sim One representou o primeiro protótipo de um corpo humano inteiro, desenvolvido por Stephen Abrahamson e Judson Denson. Em 1968, o Harvey Cardiologic Mannequin foi capaz de simular 27 condições cardíacas para o treino das habilidades médicas ${ }^{1,5}$.

Diante desta realidade, o uso de modelos de treinamento na área da saúde torna-se responsável por uma prática constante e ativa do estudante de medicina no processo ensino-aprendizagem e o desenvolvimento, cada vez mais precoce, do raciocínio clínico e sua união com a ciência básica. Ademais, verifica-se a redução dos erros médicos, bem como dos seus efeitos adversos - como consequências da utilização das tecnologias em prol da saúde humana. Nesse sentido, aspectos éticos e a necessidade de rentabilização dos recursos físicos e humanos impedem a realização dos procedimentos em pacientes reais, de modo que a prática em modelos de treinamento favoreça o aprendizado e a prática segura posteriormente na vida profissional ${ }^{3,4,6}$.

À vista disso, a aquisição dos aspectos cognitivos e psicomotores, o enfrentamento de uma situação real, objetiva e reprodutível, bem como do recebimento de um feedback por parte dos professores, possibilitam uma melhora no comportamento psicossocial e uma maior confiança no momento da ação médica propriamente dita. Assim, procedimentos invasivos ou simples podem desenvolver-se de modo mais seguro e eficaz ao paciente, deixando de expor-lhes a perigos ou aos erros médicos por imprudência, imperícia ou negligência ${ }^{1,3,4,6,7}$.

Frente a estes procedimentos, destaca-se a sondagem vesical feminina como simples, todavia capaz de desenvolver graves complicações, como infecções iatrogênicas e trauma uretral ${ }^{8}$. Baseado nesta realidade rotineira da área da saúde e na necessidade de práticas constantes, com custo reduzido e aplicação direta no currículo dos estudantes de medicina, tem-se como objetivo realizar uma revisão integrativa da literatura científica, com vistas à apresentação e à análise do desenvolvimento e dos modelos de treinamento para a sondagem vesical feminina e seus benefícios para o aperfeiçoamento das habilidades médicas.

\section{Método}

Refere-se a uma revisão integrativa da literatura científica (RI) sobre a sondagem vesical feminina e seus modelos de treinamento, baseando-se nas etapas: seleção da pergunta temática (elaboração da questão-guia), determinação dos critérios de inclusão e exclusão, triagem dos artigos (seleção da amostra), avaliação dos estudos incluídos, interpretação dos resultados e apresentação da revisão e síntese do conhecimento. Assim sendo, enquadra-se na Prática Baseada em Evidências, de modo a possibilitar uma análise crítica dos estudos e a implementação das suas evidências da prática médica. 
O levantamento dos artigos indexados correspondeu aos anos de 2000 a 2017, e deu-se no período entre novembro de 2017 e janeiro de 2018, pela consulta do PubMed e da Biblioteca Virtual em Saúde (BVS), a partir das bases de dados SciELO (Scientific Electronic Library Online), Medline (Medical Literature Analysis and Retrieval System Online) e a Lilacs (Literatura Latino-Americana e do Caribe em Ciências da Saúde). Foram utilizados os Descritores das Ciências da Saúde (DeCS) nas línguas portuguesa, inglesa e espanhola: Cateterismo urinário (Urinary atheterization; Cateterismo urinario), Feminino (female; femenino), Treinamento por simulação (Simulation training; Entrenamiento simulado) e Educação médica (Education, Medical; Educación Médica). Por fim, os descritores foram unidos pelo operador booleano "AND".

Foram utilizados como critérios de inclusão: a indexação dos artigos nas respectivas bases de dados; vínculo direto com os descritores; artigos completos e com resumos disponíveis em português, inglês e espanhol. Assim, os critérios de exclusão compreendem os estudos sem pertinência com a referida temática e não-indexados nas bases de dados. Nesse interim, a coleta dos dados foi realizada por 2 pesquisadores, independentemente, para posterior avaliação e confronto dos resultados encontrados.

A associação dos descritores encontrou 20 referências no PubMed e 21 publicações na Biblioteca Virtual em Saúde (BVS). Após a análise crítica dos artigos, bem como a computação única dos artigos duplicados e a exclusão daqueles que não coincidem com os critérios de inclusão, selecionaram-se 9 artigos, sendo esta a amostra final desta revisão integrativa da literatura científica (Tabela 1).

Tabela 1. Método de busca eletrônica nas bases de dados, de 2000 a 2017.

\begin{tabular}{lccc}
\multicolumn{1}{c}{ Base de dados } & PubMed & Biblioteca Virtual em Saúde & Total \\
Descritores & $\begin{array}{c}\text { urinary catheterization; } \\
\text { training; female; simulation }\end{array}$ & $\begin{array}{c}\text { urinary catheterization; training } \\
\text { model; female; simulation }\end{array}$ & \\
\hline Artigos encontrados $\mathrm{n}$ & 20 & 21 & 41 \\
\hline Artigos selecionados $\mathrm{n}$ & 9 & 5 & 14 \\
\hline Amostra $\mathrm{n}$ & 8 & 1 & $\mathbf{9}$ \\
\hline
\end{tabular}

\section{Resultados e discussão}

A partir da análise dos 9 artigos selecionados, verificou-se que todos (100\%) estão relacionados à área da medicina, com predomínio da língua inglesa $(66,6 \%)$, seguida da língua espanhola (33,3\%). Todas as publicações (100\%) são internacionais, relativas aos países: China (33,3\%), Estados Unidos da América (22,2\%), Espanha (22,2\%), Irlanda (11,1\%) e Dinamarca (11,1\%) (Tabela 2).

Nesse sentido, a partir dos métodos de avaliação dos artigos, tornou-se viável a interpretação e o agrupamento das informações em categorias temáticas, sendo estas: simulação em manequim low-tech (33,3\%), simulação em manequim high-tech (22,2\%), modelo porcino de simulação $(33,3 \%)$ e grau de confiança para a prática médica (55,5\%) - sendo este último uma consequência da simulação para o desenvolvimento da segurança para a realização do procedimento pós-treinamento simulado (Gráfico 1).

\section{Simulação em manequins low-tech}

O estudo de Todse et al. ${ }^{9}$ externou a importância da simulação do cateterismo vesical em manequins, a partir de cursos de habilidades clínicas, bem como da necessidade da aplicação desta na grade curricular nas escolas de medicina. Seu estudo demonstrou que os participantes garantiram resultados acima do esperado, no que tange ao sucesso na realização do procedimento com pacientes reais - denominado de teste de transferência - quando haviam recebido um treinamento de habilidades, com 


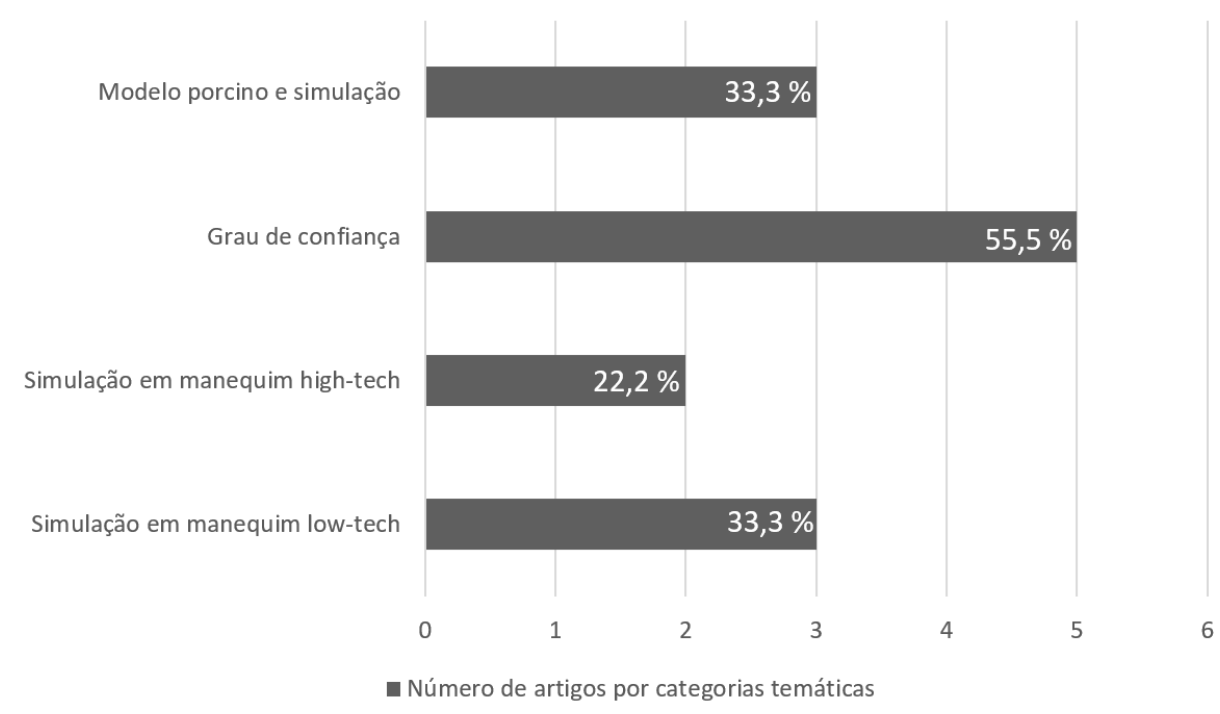

Gráfico 1. Quantidade de artigos por categoria temática.

acompanhamento de professores, padrões de avaliação de competências e feedback para cada aluno. Diante de tal realidade, identifica-se que a simulação repetitiva nos manequins de habilidades médicas possibilita a redução da iatrogenia e dos traumas uretrais após a sondagem vesical feminina.

De acordo com os estudos de Cohen et al. ${ }^{10}$, a inexistência de simuladores de cateterismo urinário de alta fidelidade e com capacidade de provocar desconforto no manequim-paciente, expõe a necessidade de treinamentos mais avançados e um desenvolvimento curricular baseado em práticas urológicas mais frequentes durante o curso de medicina. No entanto, o uso de simuladores low-tech, associado a sessões didáticas de ensino e pesquisa, além de práticas em pacientes reais - de acordo com os aspectos éticos determinados - demonstraram o aumento do grau de confiança dos estudantes, assim como das habilidades e conhecimentos inerentes à sondagem vesical masculina e feminina.

Assim sendo, confirmam-se os resultados verificados por Arriaga-González et al. ${ }^{11}$, tendo em vista que este ressaltou a influência da simulação para o melhor desenvolvimento das habilidades clínicas, da relação médico-paciente e da rapidez na tomada de decisões.

\section{Simulação em manequins high-tech}

Waters et al. ${ }^{12}$ analisou a aquisição e a retenção do conhecimento teórico-prático acerca do cateterismo urinário, por meio da utilização de Advanced Human Patient Simulator (AHPS) entre estudantes de medicina. Este estudo baseou-se na avaliação de 3 grupos: didático - os alunos receberam somente aulas teóricas; observação - os estudantes acompanhavam os médicos urologistas na prática do cateterismo em manequins - e interativo, no qual o aluno era o autor principal do processo ensino-aprendizado, por meio da prática em modelos high-tech, do conteúdo ministrado anteriormente. Vale ainda ressaltar que todos os grupos receberam treinamento laparoscópico, tendo em vista a importância da avaliação da destreza das mãos, das habilidades de inserção dos cateteres nas vias de acesso, assim como da utilização e economia dos movimentos. Frente ao grupo interativo, foi perceptível o aumento dos níveis de confiança, concomitantemente à redução do tempo de realização do cateterismo urinário.

Perante à importância dos modelos tecnológicos, Zhong et al. ${ }^{13}$, desenvolveu um novo simulador transparente do trato urinário, com o objetivo de mostrar ao aluno de medicina os procedimentos que ocorrem no interior dos órgãos, facilitando a 
compreensão das etapas processuais do cateterismo. Para tal, desenvolveu um simulador tridimensional, a partir dos softwares e de uma máquina de controle numérico computadorizada. Este modelo apresentou uma camada externa de metacrilato de polimetilo, com o intuito de dar forma ao trato urinário e o acrilonitrilo butadieno estireno, na camada interna, simulando o inchaço do trato urinário. Ademais, foi aplicado um questionário antes e depois do treinamento entre o grupo experimental e o grupo controle - que utilizou os modelos de treinamento em plástico opaco. Nesse interim, Zhong et al. ${ }^{13}$ evidenciou que, embora o conhecimento teórico de ambos os grupos seja similar, as habilidades práticas das referidas cirurgias urológicas - como a esterilização, a manipulação dos instrumentos e a inserção dos cateteres - foram mais bem desenvolvidas pelos alunos que utilizarem o novo modelo de simulação.

Logo, a simulação de um ambiente clínico representativo da realidade aumenta a aquisição e a retenção dos conhecimentos vivenciados na vida acadêmica, o que possibilitará uma boa prática na vida profissional.

\section{Modelo porcino de simulação}

O trabalho de Zhong et al. ${ }^{13}$ propôs a avaliação das habilidades adquiridas pelos estudantes de medicina após o treinamento no modelo transparente do trato urinário, em 4 porcos Yorkshire. Baseando-se nesta estratégia, foi possível analisar o desempenho a partir de 5 critérios anteriormente estabelecidos: inspeção e lubrificação do instrumento, esterilização, manuseio correto dos instrumentais, técnica de cateterismo e cistoscopia e fixação do tubo de urina e do stent retrógrado duplo-J.

Balén et al. ${ }^{14}$ também utilizou o modelo experimental para o desenvolvimento das práticas cirúrgicas durante o curso de medicina. Nesse contexto, ao descrever a anatomia do porco, verificou que somente as fêmeas poderiam ser utilizadas com vistas ao cateterismo urinário, devido às peculiaridades da uretra masculina que impedem a simulação deste procedimento.

Tal resultado também foi encontrado por $\mathrm{Ni}$ et al. ${ }^{15}$ por meio do desenvolvimento de um modelo animal de baixo custo para a cirurgia laparoendoscópica de uma única via de anastomose uretrovascular. A partir dos seus resultados, evidencia-se que a anatomia da pelve do porco é similar a dos humanos, ademais o colo uterino do porco possui a mesma consistência da uretra humana - possibilitando a simulação da uretra como via de passagem durante a sondagem vesical, sendo que a bexiga poderia ser simulada pelo íleo do referido animal. Logo, constatou-se que o modelo feminino de porco é viável e econômico para a simulação.

\section{Grau de confiança para a prática médica}

A capacidade de enfrentar os desafios diante de uma situação real define a confiança. Diante disso, Rodríguez-Díez et al. ${ }^{16}$ avaliou o grau de confiança dos estudantes de medicina, antes e após o treinamento em simuladores de sondagem vesical masculina e feminina, bem como do toque retal. Diferentemente dos resultados encontrados por Waters et al. ${ }^{12}$, no qual o grau de confiança aumentou após a realização do OSCE (Objective Structured Clinical Examination), Rodríguez-Díez et al. ${ }^{16}$ constatou tal realidade pela aplicação de um questionário separado por sexo e baseado na escala numérica Linkert, anterior e posteriormente à prática em simuladores low-tech ${ }^{2}$. Frente a isso, verificou-se que antes do treinamento, ambos os sexos apresentavam uma baixa confiança para realizar o procedimento, todavia após o treino, os valores aumentaram substancialmente, principalmente entre as mulheres. Vale ressaltar que ambos os autores relataram a importância do feedback na redução da curva de aprendizagem e do uso de simuladores para a prática de sondagem vesical.

Diferentemente deste estudo, Cohen et al. ${ }^{10}$ identificou que o grau de confiança dos estudantes de medicina, após a sessão didática e a sessão prática nos simuladores, foi igual em ambos os sexos. Vale ressaltar que Cohen et al. ${ }^{10}$ e Zhong et al. ${ }^{13}$ 
Tabela 2. Descrição dos estudos em relação à origem, língua estrangeira e área de atuação.

\begin{tabular}{lcccc} 
Origem & Língua estrangeira & Área de atuação & Número de artigos & Percentagem \\
China & Inglês & Medicina & 3 & $33,3 \%$ \\
\hline Estados Unidos & Inglês & Medicina & 2 & $22,2 \%$ \\
\hline Espanha & Espanhol & Medicina & 2 & $22,2 \%$ \\
\hline Irlanda & Inglês & Medicina & 1 & $11,1 \%$ \\
\hline Dinamarca & Inglês & Medicina & 1 & $11,1 \%$ \\
\hline
\end{tabular}

constataram que o treino baseado nas técnicas básicas de esterilidade, nos princípios de inserção dos cateteres, do conhecimento sobre o gel lubrificante, da abertura de pacotes de cateteres de forma estéril e da localização destes instrumentos no hospital, aumentaram o grau de confiança frente à realização da sondagem vesical. Ademais, corroborando com Nathwany et al. ${ }^{17}$, um ambiente simulado de ensino proporciona intervenção, avaliação e melhora, a curto e longo prazos, da capacidade e aprendizado dos estudantes.

Frente a essas categorias temáticas, verifica-se a importância da realização de uma revisão integrativa embasada nas evidências científicas para uma melhor compreensão do referido tema. Tendo em vista o rigor na análise de dados e a formulação de categorias temáticas, pode-se diminuir os vieses e fundamentar o conhecimento científico.

Nesse contexto, identifica-se a necessidade de desenvolvimento de modelos de treinamento mais acessíveis e de baixo custo, com vistas à aquisição pela maior parte das escolas médicas e ampliando sua rede de abrangência. Ressalta-se ainda a relevância de uma maior produção cientifica acerca da sondagem vesical feminina, com envolvimento de instituições de ensino e de hospitais, objetivando-se melhoras nos cuidados e uma prática profissional intrinsecamente associada às evidências e às tecnologias em prol da saúde da população.

\section{Conclusão}

A aprendizagem baseada em simulação apresenta uma importância significativa no que tange à aquisição de conhecimento teórico-prático de forma repetitiva e avaliada por profissionais, por meio do feedback. Diante desta situação, simuladores low-tech e high-tech e modelos de treinamento experimentais são capazes de desenvolver as habilidades clínicas processuais e preparar o estudante de medicina para enfrentar as situações reais, com grau de confiança e destreza manual mais desenvolvidas, em relação à prática isolada da teoria. Assim, erros iatrogênicos, traumas uretrais ou infecção do trato urinário podem ser evitados com uma prática constante nos simuladores de sondagem vesical feminina.

\section{Referências}

1. Cooper JB, Taqueti VR. A brief history of the development of mannequin simulators for clinical education and training. Qual Saf Health Care. 2004. Oct;13(Suppl 1):11-8. https://doi.org/10.1136/qhc.13.suppl_1.i11.

2. Camilo H. A educação médica baseada na simulação e em simuladores. Essências Educare. 2008 Jul;8.

3. Macieira LMM, Teixeira MDCB, Saraiva JMA. Simulação médica no ensino universitário de pediatria medical. Rev Bras Educ Med. 2017;41(1):86-91. http://dx.doi.org/10.1590/1981-52712015v41n1rb20160032.

4. Mendonça VF, Custódia EM. Nuances e desafios do erro médico no Brasil: as vítimas e seus olhares. Rev Bioét. (Impr.). 2016; 24 (1): 136-46. http://dx.doi.org/10.1590/1983-80422016241115.

5. Singh H, Kalani M, Acosta-Torres S, El Ahmadieh TY, Loya J, Ganju A. History of simulation in medicine: from resusci annie to the ann myers medical center. Neurosurgery. 2013 Oct;73(Suppl 1):S9-S14. https://doi.org/10.1227/NEU.0000000000000093.

6. Flores CD, Bez MR, Bruno RM. O uso de simuladores no ensino da medicina. RBIE. 2014;22(2):3-12. 
7. Mariani AW, Fernandes PMP. Ensino médico: simulação e realidade virtual. Diagn Tratamento. 2012;17(2):47-8.

8. Balduino LSC, Torres GV, Araújo RO, Vasconcelos QLDAQ, Germano RM, Menezes RMP. Analyzing the context of the teaching-learning process in urinary catheterization: integrative review of literatura. J Nurs UFPE on line. 2012 Sept;6(9):22507. http://dx.doi.org/10.5205/reuol.2570-20440-1-LE.0609201231

9. Todsen T, Henriksen MV, Kromann CB, Konge L, Eldrup J, Ringsted C. Short and long-term transfer of urethral catheterization skills from simulation training to performance on patients. BMC Med Educ. 2013;13(1):13-29. http://dx.doi.org/10.1186/14726920-13-29. PMid:23433258.

10. Cohen A, Nottingham C, Packiam V, Jaskowiak N, Gundeti M. Attitudes and knowledge of urethral catheters: a targeted educational intervention. BJU Int. 2016;118(4):654-9. http://dx.doi.org/10.1111/bju.13506. PMid:27104479.

11. González-Arriaga CR, González Lubcke E, Sierra-Basto G. Instalación de sonda vesical posterior a la intervención educativa en un modelo de simulación. Estudio comparativo en modelo de simulación y pacientes reales. Estudio piloto. Inv Ed Med. 2013;2(7):135-9. http://dx.doi.org/10.1016/S2007-5057(13)72702-9.

12. Waters PS, McVeigh T, Kelly BD, et al. The acquisition and retention of urinary catheterisation skills using surgical simulator devices: teaching method or students traits. BMC Med Educ. 2014;14(1):264. http://dx.doi.org/10.1186/s12909-014-0264-3. PMid:25527869.

13. Zhong $\mathrm{X}$, Wang $\mathrm{P}$, Feng J, Hu W, Huang C. Novel transparent urinary tract simulator improves teaching of urological operation skills at a single institution. Urol Int. 2015;95(1):38-43. http://dx.doi.org/10.1159/000375129. PMid:25720440.

14. Balén EM, Sáez MJ, Cienfuegos JA, et al. Anatomía del cerdo aplicada a la experimentación en cirugía general. Cir Esp. 2000;67(6):586-93.

15. Ni D, Ma X, Li H, et al. Urethrovesical anastomosis training in an economical porcine model. Urol Int. 2014;92(1):89-94. http://dx.doi.org/10.1159/000351003. PMid:23886884.

16. Rodríguez-Díez MC, Díez N, Merino I, Velis JM, Tienza A, Robles-García JE. La simulación mejora la confianza de los estudiantes para adquirir competencias en urología. Actas Urol Esp. 2013;38(6):367-72. http://dx.doi.org/10.1016/j. acuro.2013.10.007. PMid:24332529.

17. Nathwani JN, Law KE, Witt AK, Ray RD, DiMarco SM, Pugh CM. A simulation-based, cognitive assessment of resident decision making during complex urinary catheterization scenarios. Am J Surg. 2017;213(4):622-6. http://dx.doi.org/10.1016/j. amjsurg.2017.01.007. PMid:28089342.

\section{Contribuição dos autores}

Lêda Lima da Silva participou da concepção participou da concepção e desenvolvimento da pesquisa, da elaboração do desenho metodológico, da coleta e interpretação dos dados, da redação do manuscrito. Alyne Condurú dos Santos Cunha participou da concepção e desenvolvimento da pesquisa, da elaboração do desenho metodológico, da coleta e interpretação dos dados, da redação do manuscrito. Alan Luz Tembra participou da concepção e desenvolvimento da pesquisa, da elaboração do desenho metodológico e do levantamento da literatura. Geovanna Mourão Pantoja participou da elaboração do desenho metodológico, do levantamento da literatura e da redação do manuscrito. Charles Alberto Villacorta de Barros participou da revisão crítica do manuscrito. Mauro de Souza Pantoja participou com a revisão crítica do manuscrito. 